\title{
MultiModal Wireless Sensor Networks based on Wake-up Radio Receivers: An analytical Model for Energy Consumption
}

\author{
Redes Inalámbricas MultiModales de Sensores basadas en Receptores de Radio Wake-up: \\ Un modelo analítico para consumo de energía
}

\author{
Juan Aranda (iD ${ }^{1,2}$, Mario Schölzel (iD) ${ }^{3}$, Diego Mendez (iD) ${ }^{2}$, Henry Carrillo (iD) 2 \\ ${ }^{1}$ Exact Sciences and Engineering School, Universidad Sergio Arboleda, Bogotá, Colombia \\ ${ }^{2}$ Department of Electronics Engineering, Pontificia Universidad Javeriana, Bogotá, Colombia \\ ${ }^{3}$ Department of Engineering, Nordhausen University of Applied Sciences, Nordhausen, Germany.
}

\section{ARTICLE INF0:}

Received: October 19, 2018

Accepted: March 08, 2019

\section{AVAILABLE ONLINE:}

April 25, 2019

\section{KEYWORDS:}

Energy efficiency, multimodal wireless sensor network, low-duty-cycling, wake-up radio

Eficiencia energética, redes inalámbricas multimodales de sensores,

low-duty-cycling, wake-up radio
ABSTRACT: Traditionally, sophisticate power-aware wake-up techniques have been employed to achieve energy efficiency in Wireless Sensor Networks (WSNs), such as low-duty cycling protocols using a single radio architecture. These protocols achieve good results regarding energy savings, but they suffer from idle-listening and overhearing issues, that make them not reliable for most ultra-low power demanding applications, especially, those deployed in hostile and unattended environments. Currently, Wake-up Radio Receivers (WuRx) based protocols, under a dual-radio architecture and always-on operation, are emerging as a solution to overcome these issues, promising higher energy consumption reduction compared to classic wake-up protocols. By combining different transceivers and reporting protocols regarding energy efficiency, multimodality in WSNs is achieved. This paper presents an energy consumption estimation model that considers the behavior and performance of wake-up protocols based on WuRx in multi-hop communications under several cases instead of traditional low-duty cycling schemes. The results show that the WuRx with addressing does not significantly reduce the energy consumption compared to WuRx without addressing. In some cases, classic low-duty cycling protocols outperform WuRx based protocols, but in most cases, it is contrariwise, giving a strong motivation for considering multi-modal approaches in WSNs.

RESUMEN: Algunas técnicas sofisticadas de ahorro de energía se han venido empleando para lograr eficiencia energética en Redes Inalámbricas de Sensores (WSNs), tales como los protocolos de bajo ciclo de trabajo enmarcados en una arquitectura de único radio. Estos protocolos logran buenos resultados con respecto al ahorro de energía, pero sufren problemas de escucha inactiva y sobreescucha, que los hacen pocos confiables en aplicaciones que demandan alto ahorro energético, especialmente aquellas implementadas en entornos hostiles y desatendidos. Actualmente, los protocolos basados en un Receptor de Radio Wake-up (WuRx), bajo una arquitectura de dos radios y una operación siempre encendida, están surgiendo como una solución para superar estos problemas, prometiendo una mayor reducción del consumo de energía en comparación con los protocolos clásicos. Al combinar diferentes transceptores y protocolos de transmisión, se logra la multimodalidad en WSNs. Este artículo presenta un modelo de energía para estimar el ahorro de energía en varias topologías de redes de saltos múltiples bajo varios casos de uso basados en WuRx en lugar de los esquemas tradicionales. Los resultados muestran que el WuRx con direccionamiento no reduce significativamente el consumo de energía en comparación con WuRx sin direccionamiento. En algunos casos, los protocolos de bajo ciclo de trabajo superan a los protocolos basados en WuRx, pero en la mayoría de los casos, sucede lo contrario, lo cual brinda una fuerte motivación para considerar enfoques multimodales en WSNs.

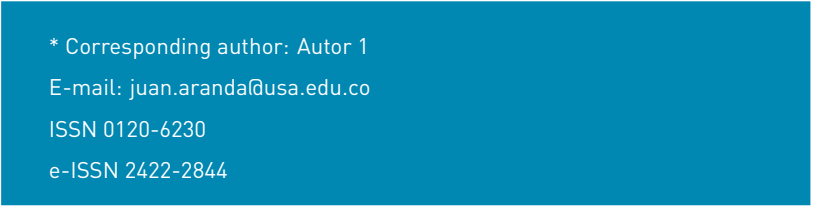

\section{Introduction}

Wireless Sensor Networks (WSNs) are widely deployed for monitoring purposes such as environmental and 
infrastructure monitoring. Typically, a WSN is composed of a large number of tiny Sensor Nodes (SNs), which are commonly battery powered and have limited energy resources. In order to save energy and to extend the lifetime of the SNs to several years, sophisticated power saving techniques must be deployed [1]. Thereby, a considerable amount of energy can be saved, while a high monitoring quality is maintained if the behavior of the SNs can be adapted dynamically to the current conditions of the system. For example, if no activity takes place in the environment, the SNs could go into sleep mode. If only spare events take place, then $\mathrm{SNs}$ may run a low-duty-cycling (LDC) protocol, while in phases of high and critical activity in the surrounding, the $\mathrm{SNs}$ should be very reactive to forward data with very low latency. Such context-aware adaptivity can be achieved in several ways, in which the transceivers and the transmission protocols play a crucial role in implementing this adaptivity and conserving energy [2].

MultiModal Wireless Sensor Networks (M2WSNs) allow using different types of transceivers and running MultiModal Switching Mechanisms (M2SMs) [3] between different protocols for reporting, depending on the current context of the environment. For instance, in some regions with high activity, the SNs start to generate sensed data that must be transmitted to the sink. Other SNs, outside of these regions, are not aware of the high activity, and they become aware of this situation by the high amount of transmissions they have to forward to the sink or by receiving an assisting message transmitted by a working node in the region [3].

Our approach considers M2WSNs where SNs have the main radio and an additional Wake-up Radio Receiver (WuRx), supporting different types of protocols. For example, all SNs can sleep until an event occurs, and when that happens, SNs are woken up by the WuRx for reporting the event to the sink. However, also all classical known LDC protocols may be executed with the main radio during some periods of activity. Thereby, we focus on the question, if there are some periods of activity, when it is beneficial regarding energy consumption to run traditional LDC protocols, instead of a wake-up protocol always using the WuRx. Consequently, the multimodality in the network is achieved by using two different transceivers and different protocols.

In this paper, we present a parameterized energy estimation model that allows us to model the behavior of particular SNs and the whole M2WSNs. We consider that this model serves as a point of comparison between wake-up protocols based on WuRx and LDC schemes regarding the power consumption. We show that WuRx with addressing will not significantly save energy compared to WuRx without addressing and that in some situations, an LDC scheme outperforms a WuRx scheme, while in some other situations, it is the other way around, giving a strong motivation for using multi-modal approaches in WSNs. Therefore, the main contribution of our work is summarized as follows: An energy consumption estimation model for MultiModal Wireless Sensor Networks that considers the behavior and performance of wake-up protocols, mainly, those based on Wake-up radio receivers. When compared to our previous work [4], this paper presents: (i) a detailed description of the proposed dual-radio layered architecture and multi-hop communications model based on WuRx for M2WSNs, (ii) an in-depth analysis of extensive simulation results that validates our proposal, and (iii) an approach to packet error and false wake-up effects in the model.

The rest of the paper is organized as follows. In Section 2 , we discuss related work regarding wake-up radio and existing energy models based on WuRx, showing that there is a significant lack of models that allow comparing the energy efficiency of WSNs with WuRx and WSNs running "classical" LDC protocols. In Section 3, the models for the proposed wake-up protocol based on WuRx and LDC schemes are introduced. Then, Section 4 presents and discusses the results under various use cases, i.e., the parameter settings for the model. Finally, we conclude the paper and propose some future work in Section 5.

\section{Related work}

Traditional MAC protocols for WSNs are based on low-duty-cycling approaches, where SNs switch between sleep and active states, and vice versa, following a predefined or on-demand scheduling [5]. LDC approaches help reducing the energy consumed by overhearing and idle listening. However, this reduction is insufficient for low-power demand WSN designs [6], where SNs are required to save energy as much as possible to extend the network's lifetime. Therefore, a Wake-up Radio Receiver (WuRX) approach has been proposed recently to overcome LDC limitations [1].

A WuRx is an ultra-low-power radio hardware, which is commonly added to $\mathrm{SNs}$ as a second radio, as shown in Figure 1. Some WuR prototypes are detailed in [2]. Typically, a WuRx is periodically listening to the channel for a pre-defined Wake-up Signal (WuS), which activates, through an interrupt signal, other electronic parts of the $\mathrm{SNs}$, for instance, the main micro-controller. The wake-up receiver can be listening for WuS in the same frequency band or at different frequencies of the main radio transceiver. The former is know as in-band channel, and the latter, out-of-band channel [2]. The in-band channel is cheaper because we can use the same main radio 
Table 1 Comparison of relevant energy models based-on WuRx schemes for WSNs.

\begin{tabular}{lllll}
\hline Authors & $\begin{array}{l}\text { Network } \\
\text { Communication }\end{array}$ & Channels & WuRx Modeling & Error Approaching \\
\hline Seyed et al.[7] & Single-hop & In-Band & Only addressing & $\begin{array}{l}\text { Bit-error rate, wake-up miss } \\
\text { and false alarm probabilities }\end{array}$ \\
\hline Lont et al. [8] & Single-hop & Out-of-Band & Only addressing & $\begin{array}{l}\text { Packet missed and false } \\
\text { wake-up probabilities }\end{array}$ \\
\hline Zhang et al. [9] & Single-hop & Out-of-Band & Only addressing & $\begin{array}{l}\text { Miss detection and false alarm } \\
\text { probabilities }\end{array}$ \\
\hline Zhang et al. [10] & Single-hop & In-Band & $\begin{array}{l}\text { Only addressing } \\
\text { Both addressing, }\end{array}$ & $\begin{array}{l}\text { None } \\
\text { non-addressing }\end{array}$ \\
\hline This work & Multi-hop & In-Band & \begin{tabular}{l} 
retransmissions) \\
\hline
\end{tabular}
\end{tabular}

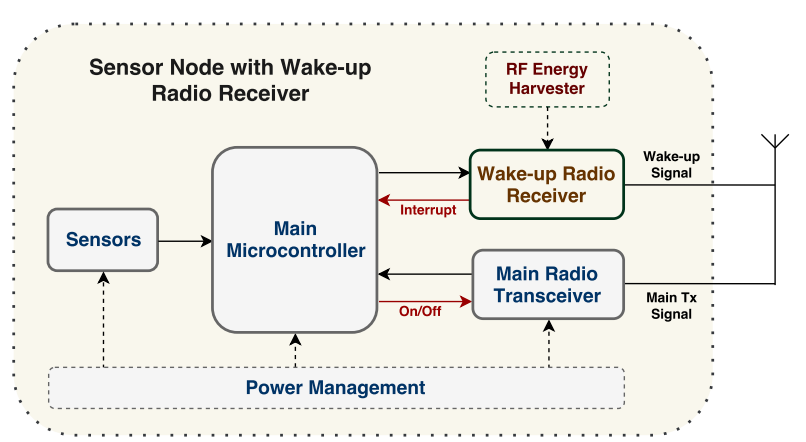

Figure 1 A generic wake-up radio receiver hardware attached to a sensor node (Based on [2]).

transceiver to transmit the WuS but at the cost of dealing with interference within the neighborhood that works at the same band. The out-of-band channel approach may decrease the interference issue, but it usually adds complexity and extra cost to the system design ltwo radio, one for WuS transmission, and another for data communication). However, compared to "classical" low-power radios, the power consumption of a WuRx is several orders of magnitude lower than these radios, allowing to keep it always-on [2]. Consequently, a WuRx can eliminate the idle-listening and waiting time issues, and help reduce the energy consumption and latency [1].

Some WuRx implementations have dedicated circuitry to perform an addressing mechanism by decoding a destination address contained in the packet header. Therefore, only the designate node is woken up instead of the entire neighborhood. This feature might allow solving the overhearing issue presented in LDC approaches. In this sense, there are mainly two manners to consider the recipient of a WuS. On the one hand, a source node can reach all the SNs within its neighborhood by a broadcast-based wake-up. All SNs within the source range receive the WuS. On the order hand, a source node intends to reach only one node within its range using dedicated circuitry. This scheme is usually known as ID-based scheme [2]. The latter is intended for selective wake-up addressing, where only the node with a specific ID is woken up. This scheme helps to reduce the false-wake-ups and the overall energy consumption of the whole M2WSNs (usually in large-scale deployments), but it requires a decoding process which is usually performed by an external micro-controller that adds an additional energy requirement to the WuRx power supply. Besides, the WuS packet needs extras bits (e.g., 2 bits [2]) for the destination address, that might require extra transmit time, hence extra energy consumption. The former can contribute to reduce the end-to-end latency, because the node does not decode the incoming WuS, but it might increase the false-wake-ups, which may be potentially costly regarding energy consumption. Therefore, there is a trade-off to be made between latency and false-wake-ups reduction.

Piyare et al. [2] made a comprehensive overview of WuRx based on MAC protocols and offered an extended taxonomy of WuRx based on routing protocols compare to Djiroun and Djenouri's work [1]. They concluded that the lifetime of the SNs could be further extended by combining WuRx capabilities with selective addressing and routing duties (e.g., T-ROME [5]) while meeting latency requirements comparable to $\mathrm{SNs}$ that use a single radio.

In Table 1, a comparison is made between relevant energy consumption models proposed in the literature and our approach. Most of the work only applies for single-hop MAC protocols and two-channel solutions. Our model takes into account multi-hop communications and the energy budget for the whole network, and the main radio and WuRx use the same channel (in-band-solution), whereby only very few works exist in that area [2]. Finally, our model allows for modeling addressing and non-addressing WuRx compared to other models that focus only on addressing. 


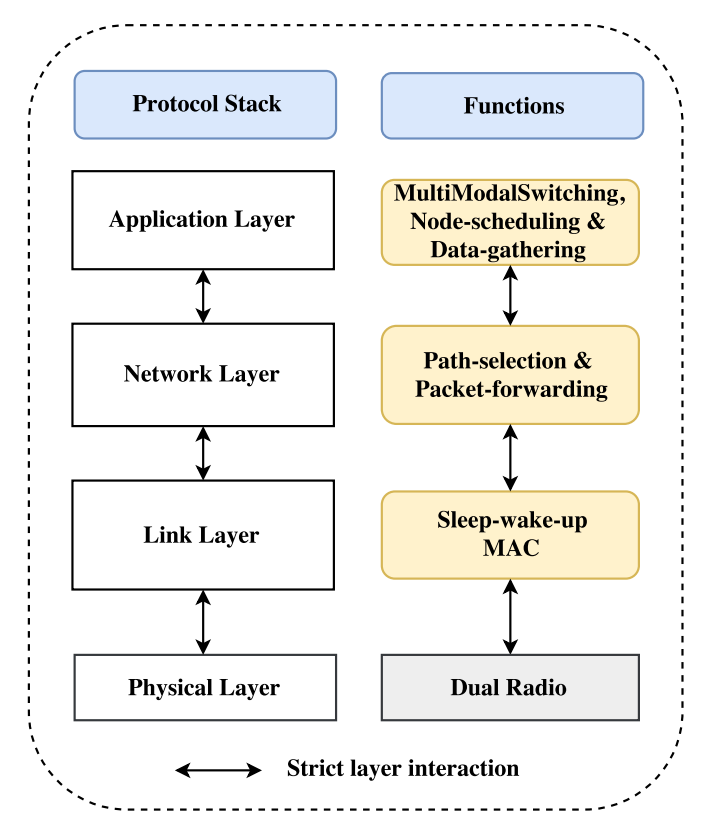

Figure 2 A layered architecture for M2WSNs in the context of monitoring applications.

\section{Energy Consumption Models}

Typically, the wake-up protocol is integrated into the link layer of a layered architecture for M2WSNs (Figure 2), which includes an application layer which runs M2SMs (e.g., eHNS [3]) using the information provided by its adjacent layer. A network layer that performs topology management duties, e.g., path selection, and packet forwarding. A link layer that executes sleep-wakeup duties combined to medium access control with retransmission functions, which aim to minimize the energy consumption at the physical layer by reducing the transmission power while providing a high monitoring quality. In this paper, we focus only on the lower-layers and consider a dual radio communication (main radio and $\mathrm{WuRx}$ ) at the physical layer, both radios sharing the same channel lin-band solution).

Figure 3 shows an example of a multi-hop communications on a tree-like topology for reporting an event and its associated data to the sink using a dual radio. A source $\mathrm{SN}$ detects an event, reports and propagates it through a known routing path towards the sink. In this schematic, the WuRx range is shorter than the main radio range. The routing path is constructed and updated by a "classical" routing protocol (e.g., RIME or RPL as done in [11]) at the network layer. Finally, the source SNs, before transmitting its sensed data towards the sink, sends a WuS packet, which wakes up all potential receivers (i.e., child and parents $\mathrm{SNs}$ ) within the wake-up radio range (i.e., those SNs that have a WuRx integrated), as shown in the right sketch on Figure 3. Later in this paper, we give more details about this operation.

The state machine diagram for such a dual radio setup of a single node is shown in Figure 4. The dashed rectangles are transition states, and the power consumption of a state, $P_{\text {State }}^{\text {component }}$, and the period a node remains in each state, $T_{\text {State/packet-type, }}^{\text {compone }}$ are shown in round brackets. The WuRx has one main state, channel - listening, and a transition state, $W u R x$ - Setup, that models its initial setup. After the setting up state, the WuRx remains listening to the channel, waiting for a WuS to arrive. Upon a WuS, the node is woken up by an external interrupt signal generated from the WuRx, as shown in Figure 1. In this case, the main radio of the node stays in its deepest sleep mode (DLPM) with the lowest power consumption $P_{D L P M}$. Otherwise, if the $\mathrm{SN}$ runs a LDC protocol without WuRx-support, some timers are needed to wake the SN up periodically (the SN stays only in a low-power mode (LPM) with power consumption $P_{L P M}$ l, and typically, $P_{L P M}$ is larger than $P_{D L P M}$.

During the active mode, the main radio remains on its Idle state, and switches between Transmitting and Receiving states depending on the task to perform: (1) to transmit a data or Ack packet to the next hop in the routing path or (2) to receive a data or Ack packet from the previous hop (to process it or to relay it). The transitions to set up the main radio are modeled in Tx Setup and $R x$ Setup states (e.g., data encapsulation), respectively. After performing these tasks, the main radio returns to its Idle state, and then to the Sleeping state, where it remains in the deepest low power mode.

In the subsequent sections, we present our underlying assumptions and briefly describe the protocol schemes for both cases, i.e., using the wake-up protocol based on the WuRx and based on the LDC scheme. Based on these descriptions, the energy model for delivering a packet over a single path to a sink is presented, which resembles already known models. Finally, these models are extended to cover the energy consumption of the whole network, based on the node density and event rates.

\subsection{Basic Assumptions}

In our analytic model and to compare the benefits of a WuRx and a LDC configuration for M2WSNs, some underlying assumptions are made:

1. The WuRx and the main radio share both the same channel. As a consequence, transmissions of the main radio are detected by the WuRx. Therefore, each SN needs, besides the main radio, only a WuRx, and not a wake-up transmitter.

2. Both radios have the same communication range. 

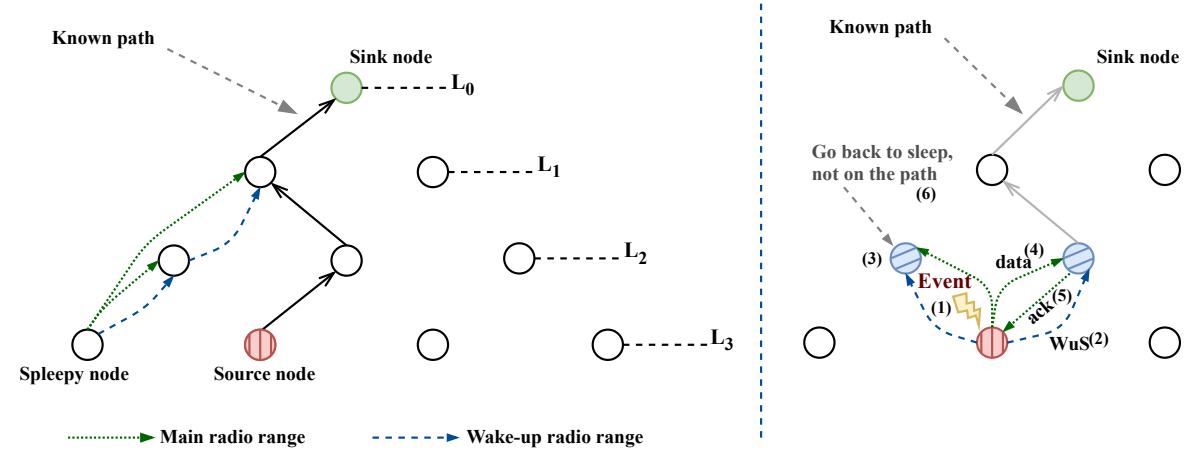

Figure 3 A dual radio multi-hop communication schematic on a tree-like topology (Based on [5]). The right sketch shows a multi-hop operation of the wake-up protocol proposed. The event data packet is propagated from the source to the sink through a known routing path previously defined.

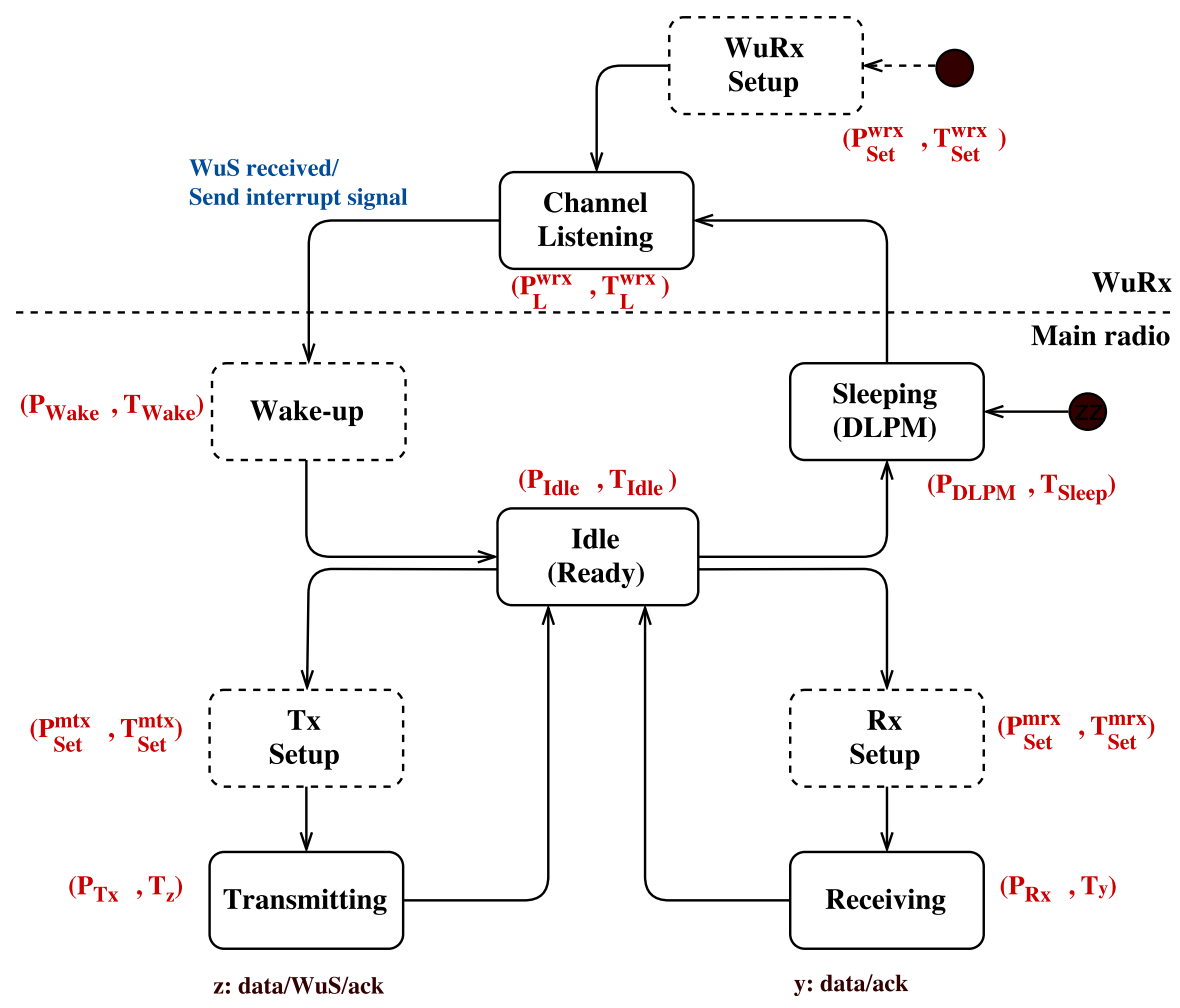

Figure 4 Dual radio state machine diagram (Based on $[7,8,12]$ ).

3. We do not care about the network topology and routing protocols. We assume that a SN knows the address of the next hop for delivering a data packet to the sink.

4. Transmitted data message is very small. Therefore, we assume that it is enclosed in the wake-up packet.

5. For the sake of simplicity, we assume that the power consumption $\left(P_{\text {node }}\right)$ of the main radio is the same for the wake-up and idle periods, receiving and transmitting a packet, and switching between states.

6. For energy estimation, we assume no packet loss during communication, i.e., an error-free channel
(Table 1).

7. All SNs sleep in LPM4 mode, i.e., the deepest operating sleep mode, until they are woken up for their active period.

\subsection{Wake-up protocol based on a WuRx scheme}

Figure 5 presents the behavior of the proposed wake-up protocol using a WuRx scheme when delivering a data packet to the sink through a known routing path. The diagram shows the packets transmitted using the main 
Table 2 List of variables.

\begin{tabular}{|c|c|}
\hline Variable & Description \\
\hline$P_{\text {Set }}^{\phi}, T_{\text {Set }}^{\phi}$ & $\begin{array}{l}\text { Power and time required to settle WuRx or } \\
\text { switch between idle and Tx (transmission) or } \\
\operatorname{Rx} \text { (Reception) states, where } \\
\phi \in\{\text { wrx } m \text { m } x, \text { mtx }\}\end{array}$ \\
\hline$P_{\text {Wake }}, T_{\text {Wake }}$ & $\begin{array}{l}\text { Power and time required for wake-up } \\
\text { procedures }\end{array}$ \\
\hline$P_{\text {Idle }}, T_{\text {Idle }}$ & Power and time consumed in idle state \\
\hline$P_{T x}, T_{z}$ & $\begin{array}{l}\text { Power and time required for } z \text { packet } T \mathrm{x}, \\
\text { where } z \in\{d a t a, a c k, W u S\}\end{array}$ \\
\hline$P_{R x}, T_{y}$ & $\begin{array}{l}\text { Power and time required for } y \text { packet } \mathrm{Rx} \text {, } \\
\text { where } y \in\{\text { data,ack }\}\end{array}$ \\
\hline$P_{D L P M}, T_{\text {Sleep }}$ & $\begin{array}{l}\text { Power and time consumed in the deepest } \\
\text { sleep state }\end{array}$ \\
\hline$P_{L P M}$ & $\begin{array}{l}\text { Power consumption of SNs in low power } \\
\text { mode (LPM) mode }\end{array}$ \\
\hline$P_{\text {node }}$ & $\begin{array}{l}\text { Power consumption of SNs when } \\
\text { transmitting, receiving, listening, switching } \\
\text { between states. }\end{array}$ \\
\hline$P_{\triangle D L P M}$ & $\begin{array}{l}\text { Power budget allocated only to the active } \\
\text { mode excluding the power budget allocated } \\
\text { to the deepest sleep mode. }\end{array}$ \\
\hline$P_{w r x}$ & $\begin{array}{l}\text { Power consumption of WuRx during its } \\
\text { operation }\end{array}$ \\
\hline$T_{s w}^{\text {sleep }}$ & $\begin{array}{l}\text { Time required to switch between active and } \\
\text { sleep states }\end{array}$ \\
\hline$N$ & Total number of SNs in the M2WSNs \\
\hline$h$ & Number of hops in the known path \\
\hline nbor & Number of neighbors that are woken up \\
\hline mbte & Mean time between two events \\
\hline$T$ & Total time duration of an active cycle \\
\hline$t_{\text {run }}$ & Total runtime of the M2WSNs \\
\hline
\end{tabular}

radio. In Table 2, we also give a list of variables and their corresponding description, which are used in the diagrams and the later analysis.

The timing diagram in Figure 5 presents the interactions between the $\mathrm{SNs}$ within the established path to the sink. The wake-up protocol operates under a transmitter-initiator scheme, where the source node (i.e., the sender node) or working node [3] starts the communication by first sending a WuS packet la dummy ack packet) using its main radio and then data packets with destination address. After sending a data packet, the sender waits for an acknowledge packet (ack) (e.g., $\left.T_{\text {Idle }}+P_{S e t}^{m r x}\right)$, and if no ack-packet is received, the sender transmits the same data packet again, until an ack-packet arrives or the number of trials is exhausted. The WuS-packet wakes up all potential receivers, i.e., those $\mathrm{SNs}$ within the interference range of the sender node las shown in Figure 3, step 3). Hence, also the non-destination $\mathrm{SN}$ s turn on their main radio, remain active until a data packet is received, but then go back to sleep because the destination address does not match their own address (refer to step 6 in Figure 3). For simplicity in Figure 5, the non-destination $\mathrm{SN}$ interactions are not shown.
The designated receiver (i.e., Hop - 1, the node within the multi-hop routing path) should receive one of the subsequent data packets, and then, send an ack-packet back to the sender. This ack-packet also serves as a WuS-packet which wakes up the next hop (i.e., Hop - 2), and $H o p-1$ can forward the data packet to $H o p-2$, right after sending the ack packet. Finally, Hop -1 goes back to sleep. This procedure is repeated throughout the hops in the known path until the data packet is delivered to the sink, as shown in Figure 5.

\section{Energy model for a single path}

Based on the assumptions made and the timing diagram of Figure 5, the energy budget, $E_{h o p}$ in equation (1), allocated to a single intermediate node (e.g., Hop - 1) on a known path is given by:

$$
\begin{aligned}
& E_{\text {hop }}=\left(T_{\text {wake }}+2 T_{\text {data }}+2 T_{\text {ack }}\right) \times P_{\Delta D L P M} \\
& +\left(4 T_{\text {Set }}^{\phi}+5 T_{\text {Idle }}+T_{\text {sw }}^{\text {sleep }}\right) \times P_{\Delta D L P M}
\end{aligned}
$$

where $P_{\triangle D L P M}=P_{\text {node }}-P_{D L P M}$ is the power consumed in active mode on top of the power consumed in DLPM.

Consequently, the energy budget allocated for transmitting a single data packet over $h$ many hops on the known path is given by the equation (2).

$$
e_{\text {path }}=(h-1) \times E_{h o p}+E_{\text {source-sink }}
$$

The first term gives the power consumption of the $h-$ 1 intermediate SNs on the path, while the second term complements the power consumption of the source and sink, $E_{\text {source-sink }}$ in equation (3), which it is detailed below:

$$
\begin{aligned}
& E_{\text {source-sink }}=\left(2 T_{\text {Wake }}+2 T_{\text {data }}+3 T_{\text {ack }}\right) \times P_{\Delta D L P M} \\
& +\left(6 T_{\text {Set }}^{\phi}+7 T_{\text {Idle }}+2 T_{\text {sw }}^{\text {sleep }}\right) \times P_{\Delta D L P M}
\end{aligned}
$$

Depending on the WuRx used (supporting addressing or non-addressing), also other SNs in the surrounding of the $\mathrm{SNs}$ on the path are woken up. In our model, we denote this number of nodes as nbor and assume that in M2WSNs with equally distributed SNs, nbor is constant for every node. If the WuRx supports addressing, then nbor $=1$, otherwise, we assume that each node on the path wakes up nbor $>1 \mathrm{SNs}$ in its surrounding. These non-destination SNs stay awake until they have received a data packet with the destination address. Afterward, SNs realize that they are not the destination and can go back to sleep. Hence, the energy consumed by these $\mathrm{SN}$ s during their active period, $E_{n o n-d s t}$, states in equation (4).

$$
\begin{gathered}
E_{\text {non-dst }}=\left(T_{\text {Wake }}+T_{\text {data }}\right) \times P_{\Delta D L P M} \\
+\left(T_{\text {Set }}^{\phi}+2 T_{\text {Idle }}+T_{\text {sw }}^{\text {sleep }}\right) \times P_{\Delta D L P M}
\end{gathered}
$$




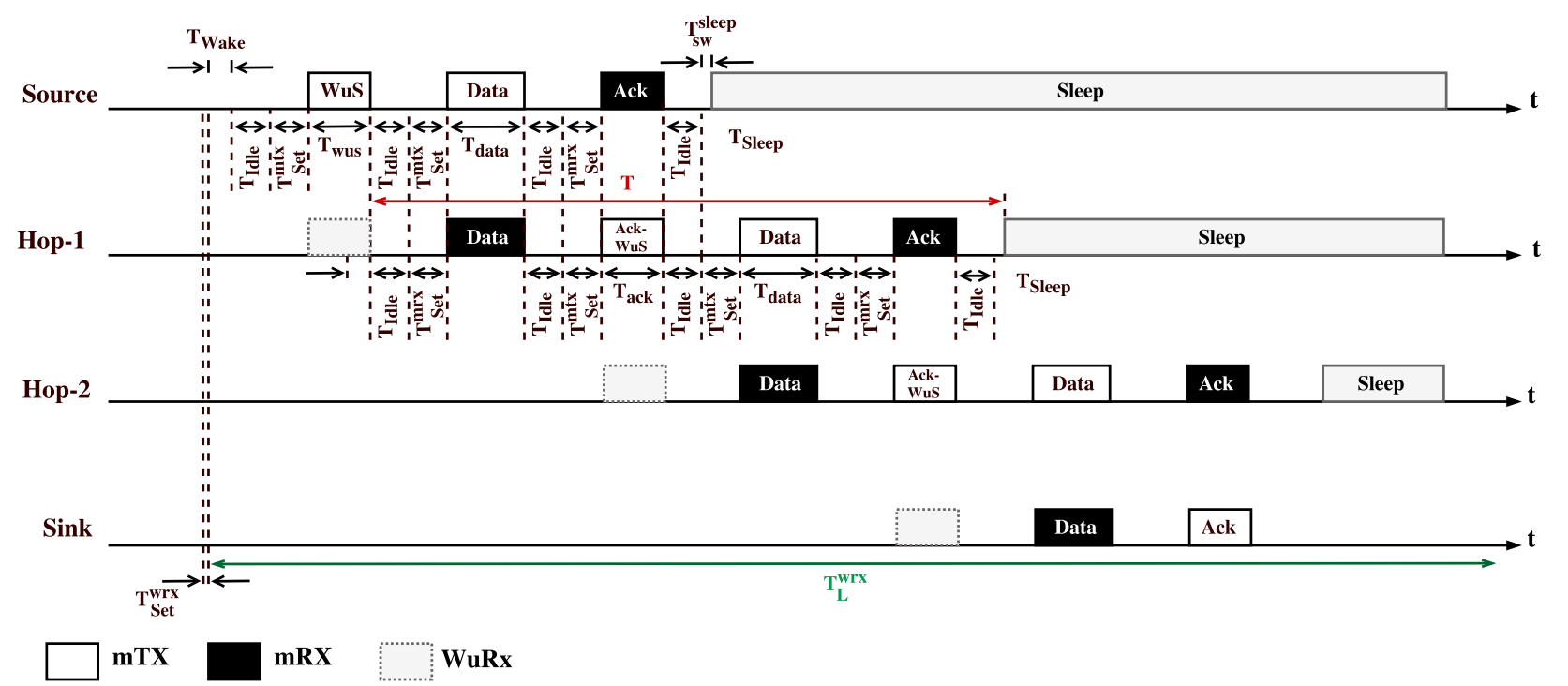

Figure 5 Timing diagram for delivering one event data packet over hops through the known path (Based on [8]) using a WuRx scheme. The non-destination SNs (not within the known path) interactions are not shown in the diagram.

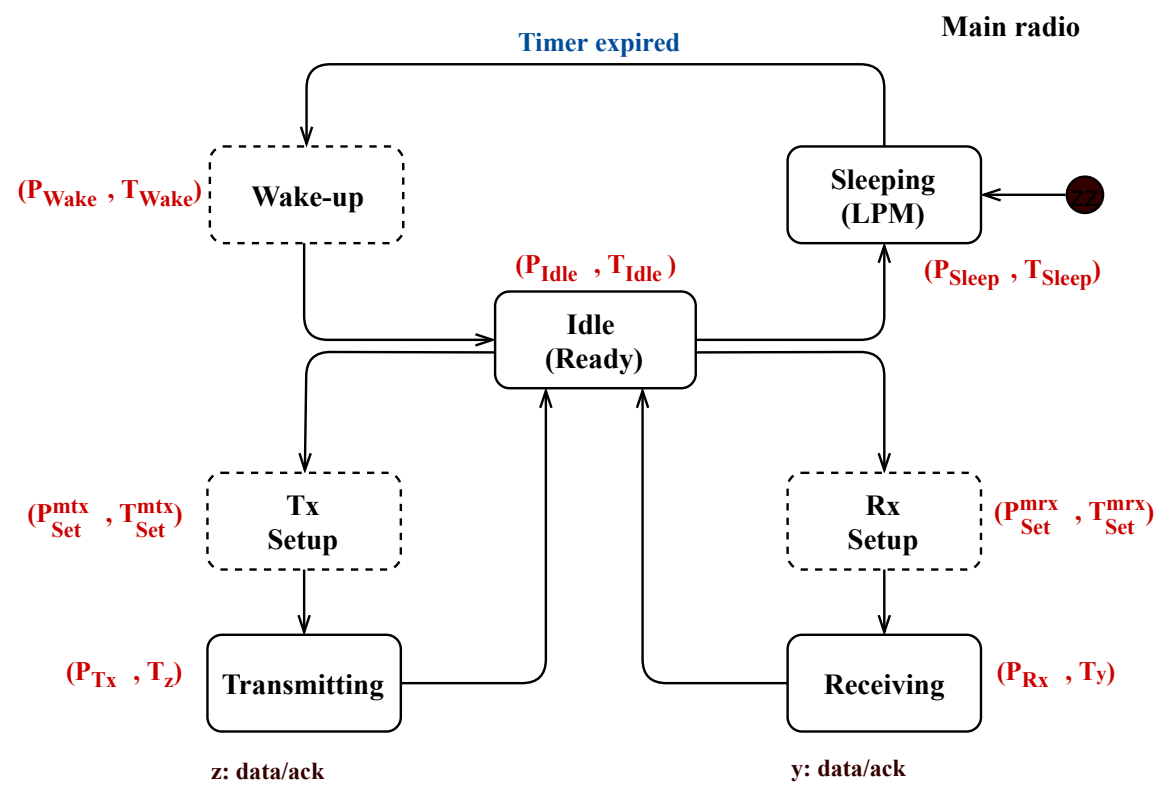

Figure 6 Single radio state machine diagram for LDC schemes.

Therefore, the total energy consumed by each event to deliver it towards the sink (only if a wake-up event occurs), $e_{\text {wakeup }_{\text {event }}}$, considering equations (2) and (4), is given by the equation (5).

$$
e_{\text {wakeup }_{\text {event }}}=e_{\text {path }}+(\text { nbor }-1) \times h \times E_{\text {non-dst }}
$$

\section{Energy model for the complete M2WSNs}

Now, we provide the model for the energy consumption of the whole M2WSNs. We divide the total energy consumption of the M2WSNs in two parts, as shown in equation (6).

$$
e_{\text {total }}=e_{\text {base }}+e_{\text {active }}
$$

The first term covers the base-energy consumption that is always present in DLPM. $e_{\text {base }}$ is the power consumed in the DLPM by each node over time and its WuRx, during the total runtime of the M2WSNs, and it is defined as shown in equation (7).

$$
e_{\text {base }}=N \times t_{\text {run }} \times\left(P_{w r x}+P_{D L P M}\right)
$$

The second term in equation (6) covers the energy consumption on top of the base-energy-consumption 
during the active period of SNs. Depending on the activity in the network, the energy budget for all events is given by the equation (8).

$$
e_{\text {active }}=\left(t_{\text {run }} / \text { mtbe }\right) \times e_{\text {wakeup }_{\text {event }}}
$$

where $t_{\text {run }} / m$ tbe is the number of events during the total runtime $\left(t_{\text {run }}\right)$.

\subsection{Wake-up protocol based on a LDC scheme}

The wake-up protocol presented in this section resembles the ideas of already existing protocols based on LDC schemes such as those proposed in [13]. The protocol follows the radio state machine introduced in Figure 4, without the states related to the WuRx, a transition between Sleeping and Wake-up states is added (which occurs after a timer expired), and use LPM instead of DLPM. Hence, the single radio state machine for LDC schemes results as shown in Figure 6.

The wake-up protocol operates under a sender-initiated LDC scheme. Every node periodically wakes up to listen to the communication channel, and to determine if there are potential incoming data packets during a $T_{\text {Awake }}$ period. If no data packet is detected, the node goes back to LPM and sleeps until its next scheduled wake-up interval le.g., $T_{\text {Sleep }}$ ), as shown in Figure 7 (i.e., refer to Hop -1 time line).

When a sender node has a data packet to transmit, it repeatedly sends a beacon to its neighbors until an ack-packet is received (every $T_{\text {Idle }}+P_{S e t}^{m t x}$ ). The beacon is the full data packet with the destination address luseful if a payload is small). Therefore, only the designated receiver (e.g., Hop - 1) acknowledges the received data packet. After receiving the ack packet, the sender node stops transmitting the data packet and goes back to the sleep period, as shown in Figure 7. This procedure is followed by each hop until the data packet is delivered to the sink.

\section{Energy model for a single path}

By following an approach similar to that of Section 3.2, we came up with the energy budget allocated for delivering an event data packet to the sink over $h$ many hops as states equation (9).

$$
e_{\text {path }}=e_{\text {hop }} \times h
$$

where $e_{\text {hop }}=e_{\text {recv }}+e_{f w d}$ is the energy budget allocated to a node to forward an event to the next hop. This energy budget is composed of two parts. The first part, $e_{\text {recv }}$, covers the energy for receiving the packet, and is given by the equation (10).

$$
e_{\text {recv }}=T_{\text {ack }} \times P_{\text {node }}
$$

Receiving takes place during the regular listen period of a node, whose energy budget is already covered by $e_{\text {base }}$ in (12). For that reason, no additional energy budget needs to be allocated, except the energy to receive the ack-packet. The second part, $e_{f w d}$, gives the energy budget required to forward the packet, which takes place usually during the regular sleep phase of receiver SNs. In the worst-case, the sender hits the active-period of the receiver after time $T_{\text {sleep }}+2 T_{\text {data }}$. In the best case, the first data packet of the sender hits the active-period of the receiver. We assume that the active period of the receiver is hit on average after half the worst-case time. Hence, $e_{f w d}$ is given by the equation (11).

$$
\begin{aligned}
& e_{\text {fwd }}=\left\{\left(T_{\text {Sleep }}+2 T_{\text {data }}\right) / 2+T_{\text {ack }}\right\} \times P_{\text {node }} \\
& +\left(T_{\text {Idle }}+T_{\text {Set }}^{\phi}\right) \times P_{\text {node }}
\end{aligned}
$$

\section{Energy model for the whole M2WSNs}

The total energy consumed by the whole M2WSNs is also composed by two part as states in (6), but the formulas for $e_{\text {base }}$ and $e_{\text {active }}$ are given by equations (12) and (13).

$$
\begin{gathered}
e_{\text {base }}=T_{\text {on }} \times P_{\text {node }}+T_{\text {off }} \times P_{L P M} \\
e_{\text {active }}=\left(t_{\text {run }} / \text { mtbe }\right) \times e_{\text {path }}
\end{gathered}
$$

where $T_{o n}$ and $T_{\text {off }}$ are the sum of the active and sleep times, respectively, of all SNs, as shown in equation (14).

$$
\begin{aligned}
& T_{\text {on }}=N \times\left(t_{\text {run }} / T\right) \times\left(T_{\text {Awake }}+T_{\text {Wake }}+T_{\text {sw }}^{\text {sleep }}\right) \\
& T_{\text {off }}=N \times\left(t_{\text {run }} / T\right) \times T_{\text {Sleep }}
\end{aligned}
$$

where $T=T_{\text {Wake }}+T_{\text {Awake }}+T_{\text {sw }}^{\text {sleep }}+T_{\text {Sleep }}$ is the duration of a whole wakeup-cycle as shown in Figure 7.

The $e_{\text {base }}$ in (12) summarizes the energy budget needed for the regular wake-up and sleep cycles, i.e., if no transmission occurs at all in the M2WSNs, then only $e_{\text {base }}$ is consumed. Meanwhile, the $e_{\text {active }}$ in (13) is the energy budget allocated to the transmission of a single event to the sink. This energy budget includes only the additional energy needed on top of $e_{\text {base }}$ for transmitting the event to the sink.

\section{Results}

Based on the proposed energy model (6), we can estimate the total power consumption for the whole M2WSNs using both schemes under different WSN configurations varying the number of $\mathrm{SNs}(N)$, event frequency (mtbe), number of hops in the known path $(h)$, and the number of woken up neighbors along the path (nbor). Thus, we perform a parameter sensitivity study for different configurations (i.e., the parameter settings for the model). Table 3 shows the values employed in the models, which are based on a 


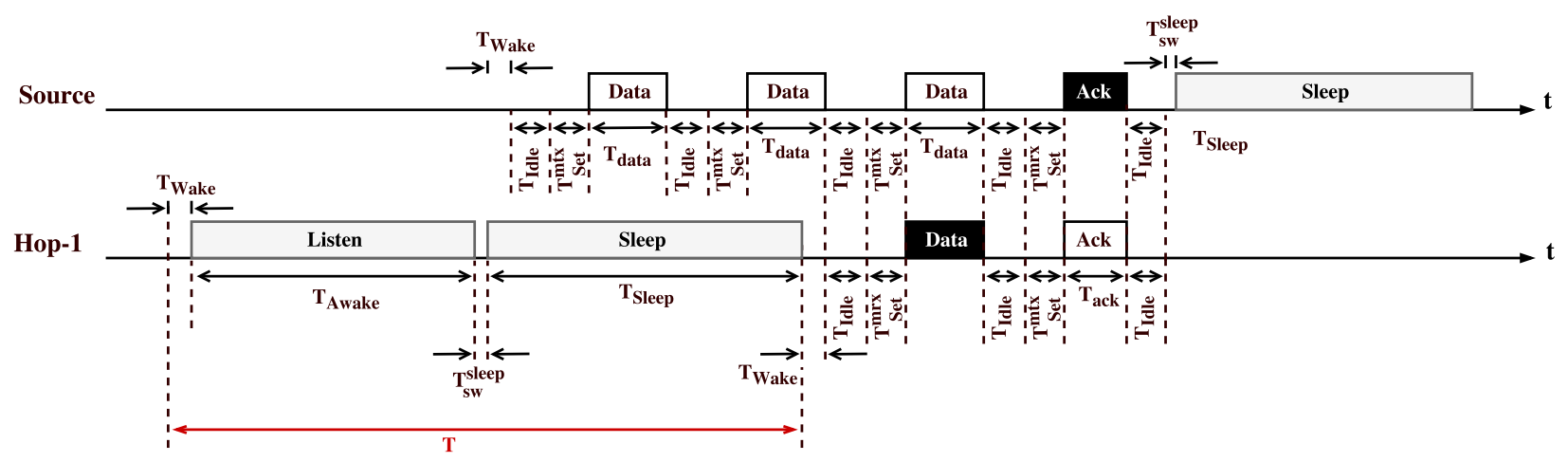

mTX mRX

Figure 7 Timing diagram for delivering one event data packet over a single hop using a LDC scheme.

Table 3 Setting parameters.

\begin{tabular}{llll}
\hline Parameters & Value & Parameters & Value \\
\hline Air data rate & $100 \mathrm{kbit} / \mathrm{s}$ & $N$ & 200 \\
$T_{\text {ack }}, T_{\text {Wake }}$ & $1.28 \mathrm{~ms}$ & $h$ & 5 \\
$T_{\text {data }}$ & $2.56 \mathrm{~ms}$ & nbor & 50 \\
$T_{\text {Sleep }}$ & $10 \mathrm{sec}$ & mtbe & $60 \mathrm{sec}$ \\
$T_{\text {Awake }}$ & $4 T_{\text {data }}$ & $t_{\text {run }}$ & 1 year \\
$T_{\text {Set }}^{\phi}$ & $1 \mathrm{~ms}$ & $T_{\text {sweep }}^{\text {slee }}$ & $5 \mu \mathrm{s}$ \\
$T_{\text {Set }}^{\text {wrx }}$ & negligible & $T_{\text {Idle }}$ & $799 \mu \mathrm{s}$ \\
$P_{\text {node }}$ & $33 m A \times 3.3 \mathrm{~V}$ & $P_{\text {wrx }}$ & $150 \mu W$ \\
$P_{D L P M}$ & $0.1 \mu A \times 3.3 \mathrm{~V}$ & $P_{L P M}$ & $6.6 \mathrm{~mA} \times 3.3 \mathrm{~V}$ \\
\hline
\end{tabular}

mote constructed with the commercial micro-controller MSP430 and transceiver C1101 at $868 \mathrm{MHz}$ along with a WuRx. This WuRx allows for permanently listening while consuming $150 \mu \mathrm{W}$.

First, we analyzed the impact on the energy consumption when considering different low-power modes supported by the node employing the WuRx, as shown in Table 4. For the first scenario, we considered the classical use case where $P_{w r x}+P_{D L P M}<P_{L P M}$, with $P_{D L P M}=0.1 \mu \mathrm{A} \times 3.3 . \mathrm{V}$ nine times lower than $P_{L P M}=9 \times P_{D L P M}$ lassuming that all peripherals can be turned off). For this scenario, varying $m$ tbe and $T_{\text {Sleep }}$ shows that a significant energy budget could be saved when this WuRx protocol is used, i.e., the ratio between WuRx and LDC power consumption ranges from 0.02 to 0.05 .

In the second scenario, we used the real $P_{w r x}$ value, where $P_{w u r x}+P_{D L P M}>P_{L P M}$. Although the power consumption of the SN using the WuRx is higher than without WuRx, using the WuRx protocol still saves $50 \%$ of the energy budget. However, the energy consumption is very sensitive to the traffic load, as shown in Table 4, where the LDC scheme becomes better than WuRx scheme for long sleep periods and low event rates. Hence, in such situations, the LDC scheme should be used instead.

In the third scenario, we assumed $P_{D L P M}=P_{L P M}=$ $6.6 m A \times 3.3 . V$, reflecting the case that the WuRx configuration cannot benefit on the SN from a DLPM, e.g., due to some peripherals that must be turned on all the time. If we employ the same LPM, both wake-up protocols cause almost the same energy consumption, being WuRx scheme a little better than LDC scheme. This scenario is also less sensitive to the network activity.

In sum, a dual radio communication becomes beneficial in scenarios of short and long sleep periods, and light and heavy event rates, when the energy consumption of the WuRx is in the order of some micro-watts, and the SN remains in its deepest low power mode (i.e., LPM4). For $P_{w u r x}+P_{D L P M} \geq P_{L P M}$, the wake-up protocol based on WuRx performs better than LDC schemes in circumstances of high event occurrence rates, due to its always-on listening mode.

The curves in Figure 8 are obtained by varying the same single parameter in both models. Besides, the study is made under the third scenario, where mtbe $=60 \mathrm{~s}$ and $T_{\text {sleep }}=10 \mathrm{~s}$. For each parameter value, the power 
Table 4 Comparison of the total energy consumption of M2WSNs under two low-power modes.

\begin{tabular}{|c|c|c|c|c|}
\hline \multirow[b]{2}{*}{ Scenario } & \multirow[b]{2}{*}{ Value $[\mu W]$} & \multicolumn{3}{|c|}{ Ratio (WuRx/LDC) } \\
\hline & & $\begin{array}{l}m t b e=60 s \\
T_{\text {Sleep }}=10 \mathrm{~s}\end{array}$ & $\begin{array}{l}m t b e=600 s \\
T_{\text {Sleep }}=10 \mathrm{~s}\end{array}$ & $\begin{array}{l}m \text { tbe }=600 \mathrm{~s} \\
T_{\text {Sleep }}=25 \mathrm{~s}\end{array}$ \\
\hline 1 & $\begin{array}{l}P_{w r x}=1 \\
P_{L P M}=2.97 \\
P_{D L P M}=0.33\end{array}$ & 0.05 & 0.02 & 0.03 \\
\hline 2 & $\begin{array}{l}P_{w r x}=150 \\
P_{L P M}=2.97 \\
P_{D L P M}=0.33\end{array}$ & 0.46 & 1.00 & 1.38 \\
\hline 3 & $\begin{array}{l}P_{w r x}=150 \\
P_{L P M}=21.8 \\
P_{D L P M}=21.8\end{array}$ & 0.99 & 1.00 & 1.00 \\
\hline
\end{tabular}
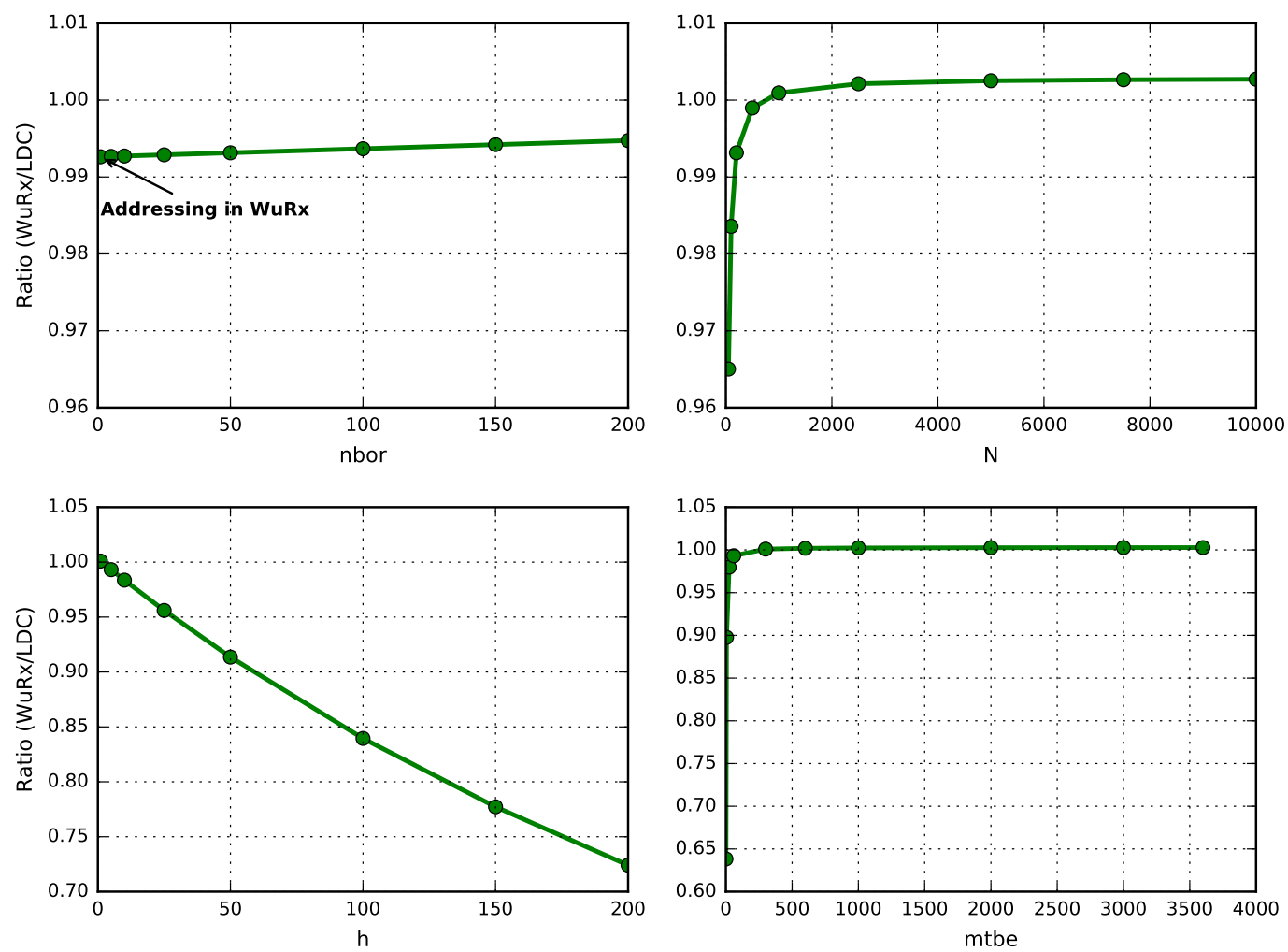

Figure $\mathbf{8}$ The ratio of the total energy consumption between both schemes under different use cases.

consumption in each model was computed, and the ratio, i.e., power consumption WuRx-based model/power consumption LDC-based model, is shown on the $y$-axis. Hence, if the ratio is less than one, the power consumption in the WuRx-based model is smaller than the LDC-based model, and vice-versa.

The effect of varying the nbor is negligible, according to the results presented in the top left graphic. When nbor $=1$ (i.e., addressing in WuRx is used) the energy consumption is almost the same as for larger nbor values (modeling no addressing). We can conclude that a broadcast-based wake-up is not energy costly compared to an ID-based wake-up when the size of the neighborhood increases and a high event rate traffic is considered. Besides, compared to a WuRx that supports a WuS decoding operation, the total power consumed for waking up the microcontroller using an interruption signal, which in turn switches on the main radio transceiver to process the incoming data packet, and then, to go back to the sleep mode, is insignificant. 
Increasing the number of SNs in the M2WSN $(N)$ has little effect, as shown in the top right graph, which implies that the total size of the M2WSN does not affect the effectiveness of a particular protocol scheme, if the other parameters are constant. However, the performance of the wake-up protocol based on WuRx decreases as $N$ increases, due to the energy consumed by non-destination $\mathrm{SNs}$ that remain awake when an event occurred and until they realize that they are not the destination node. In this situation, it might be beneficial to use a WuRx with addressing support and a false wake-ups reduction strategy.

For higher number of hops $(h)$ (third bottom left graph on Figure 8), the protocol based on WuRx performs better than based on LDC and becomes beneficial, thanks to the WuRx operations that allow SNs within the routing path to remain less time waiting for the next hop to wake up compared to LDC schemes.

Finally, for short event periods (mtbe). WuRx scheme is better, but somewhat affected by long event periods, due to the always-on operation assumed for the WuRx.

\section{Conclusions and Future Work}

In this paper, we presented an energy consumption estimation model that considers the behavior and performance of wake-up protocols based on Wake-up Radio Receivers (WuRx) and the traditional low-duty-cycling (LDC) schemes employed in M2WSNs and multi-hop communications.

M2WSNs based on an always-on ultra-low-power WuRx schemes save significantly more energy compared to "classic" LDC schemes when using many hops on the routing path and for short event periods. Besides, the WuRx with addressing does not significantly save energy compared to WuRx without addressing, under a traffic load with low event rates. However, for circumstances of long event rates, the WuRx scheme might lose against an LDC approach regarding idle-listening. Duty-cycling the WuRx could be considered as a possible solution to this issue.

In the energy consumption model based on WuRx, we considered the worst case, i.e., where all SNs within a neighborhood into the M2WSNs are woken-up when a source node has an event packet to transmit towards the sink. Therefore, the energy consumed due to false wake-up is implicitly considered in the model, i.e., equation (4). The expectation of successful event-packet transmitted to the sink can be modeled as a factor, which follows a distribution probability. This factor can be introduced into equation (2).
Therefore, as future work, we propose to include the effect of packet error losses into the model. Besides, model validation is required under emulations and laboratory-based experiments. In addition, we expect to incorporate the results into a multimodal switching scheme to dynamically select the appropriate wake-up protocol based on the circumstance, getting the best of both approaches (i.e., WuRx and LDC).

\section{Acknowledgements}

The authors would like to acknowledge the cooperation of all partners within the Auto-configurable, self-healing distributed wireless sensor networks supporting high dependability for critical applications project. The authors would also like to thank all the institutions that supported this work: Pontificia Universidad Javeriana (Project ID: 5685), the German Research Foundation (Deutsche Forschungsgemeinschaft - DFG) (Project ID: KR 3576/21-1), the Colombian Administrative Department of Science, Technology and Innovation (Colciencias) (Project ID: FP44842-090-2015), and the Universidad Sergio Arboleda (Project ID: IN.BG.086.17.003/OE4).

\section{References}

[1] F. Z. Djiroun and D. Djenouri, “MAC protocols with wake-up radio for wireless sensor networks: A review," IEEE Communications Surveys Tutorials, vol. 19, no. 1, pp. 587-618, 2017.

[2] R. Piyare, A. L. Murphy, C. Kiraly, P. Tosato, and D. Brunelli, “Ultra low power wake-up radios: A hardware and networking survey," IEEE Communications Surveys Tutorials, vol. PP, no. 99, pp. 1-1, 2017.

[3] J. Aranda, H. Carrillo, and D. Mendez, “Enhanced multimodal switching mechanisms for node scheduling and data gathering in wireless sensor networks," in 2017 IEEE Colombian Conference on Communications and Computing (COLCOM), Aug 2017, pp. 1-6.

[4] J. Aranda, M. Schölzel, D. Mendez, and H. Carrillo, “An energy consumption model for multimodal wireless sensor networks based on wake-up radio receivers," in 2018 IEEE Colombian Conference on Communications and Computing (COLCOM), May 2018, pp. 1-6.

[5] T. Kumberg, M. Schink, L. M. Reindl, and C. Schindelhauer, "T-ROME: A simple and energy efficient tree routing protocol for low-power wake-up receivers," Ad Hoc Networks, vol. 59, pp. 97-115, 2017.

[6] J. Oller, I. Demirkol, J. Casademont, J. Paradells, G. U. Gamm, and L. Reindl, "Has Time Come to Switch from Duty-Cycled MAC Protocols to Wake-Up Radio for Wireless Sensor Networks?" IEEE/ACM Transactions on Networking, vol. 24, no. 2, pp. 674-687, 2016.

[7] N. Seyed Mazloum and O. Edfors, “Influence of Duty-Cycled Wake-Up Receiver Characteristics on Energy Consumption in Single-Hop Networks," IEEE Transactions on Wireless Communications, vol. 16, no. 6, pp. 3870-3884, 2017.

[8] M. Lont, D. Milosevic, P. G. M. Baltus, A. H. M. Van Roermund, and G. Dolmans, "Analytical models for the wake-up receiver power budget for wireless sensor networks," GLOBECOM - IEEE Global Telecommunications Conference, 2009.

[9] Y. Zhang, L. Huang, G. Dolmans, and H. de Groot, “An analytical model for energy efficiency analysis of different wakeup radio schemes," 2009 IEEE 20th International Symposium on Personal, Indoor and Mobile Radio Communications, pp. 1148-1152, 2009. 
[10] M. Zhang, D. Ghose, and F. Y. Li, “Does wake-up radio always consume lower energy than duty-cycled protocols?" in 2017 IEEE 86th Vehicular Technology Conference (VTC-Fall), Sept 2017, pp. 1-5.

[11] R. Piyare, T. Istomin, and A. L. Murphy, "WaCo: A Wake-Up Radio COOJA Extension for Simulating Ultra Low Power Radios," in Proceedings of the 2017 International Conference on Embedded Wireless Systems and Networks. USA: Junction Publishing, 2017. pp. 48-53.
[12] M. Al Ameen, N. Ullah, M. S. Chowdhury, S. R. Islam, and K. Kwak, "A power efficient MAC protocol for wireless body area networks," EURASIP Journal on Wireless Communications and Networking, vol. 2012, no. 1, p. 33, 2012.

[13] M.-P. Uwase, M. Bezunartea, J. Tiberghien, J.-M. Dricot, and K. Steenhaut, "Experimental Comparison of Radio Duty Cycling Protocols for Wireless Sensor Networks," IEEE Sensors Journal, vol. 17, no. 19, pp. 6474-6482, 2017. 